\title{
Pitch discrimination in the early blind
}

\section{People blinded in infancy have sharper listening skills than those who lost their sight later.}

D. o blind people develop superior abilities in auditory perception to compensate for their lack of vision? They are known to be better than sighted people at orientating themselves by sound, but it is not clear whether this enhanced awareness extends to other auditory domains, such as listening to music or to voices. Here we show that blind people are better than sighted controls at judging the direction of pitch change between sounds, even when the speed of change is ten times faster than that perceived by the controls - but only if they became blind at an early age. The younger the onset of blindness, the better is the performance, which is in line with cerebral plasticity being optimal during the early years.

Auditory spatial localization is enhanced in early-blind subjects ${ }^{1-3}$, but the effect of blindness on performance in the nonspatial auditory domain is less clear: blind subjects seem to be better at some tasks, such as the perception of chords, than others ${ }^{4-7}$. Age at the onset of blindness, which could be an important factor, has not generally been considered.

We compared the performance of a group of early-blind subjects $(n=7$; ages, 21-40 years; onset of blindness, $0-2$ years after birth), a group of late-blind subjects ( $n=7$; ages, 24-46; onset of blindness, 5-45 years) and a group of sighted controls ( $n=12$; ages, $21-37)$ in judging the direction of pitch change (for details, see supplementary information). In the task, which is based on psychophysical testing of sighted individuals $^{8,9}$, subjects hear two pure tones of different frequencies at each trial and have to decide whether the pitch is rising (second sound with higher pitch) or falling. In the reference condition (ST in Fig. 1), the pitch difference was one-eighth of an octave (1.5 semitones, or 150 cents) and the duration of each tone was 333 milliseconds (Fig. 1a).

Task difficulty was parametrically manipulated in both the temporal and spectral domains, either by successively dividing tone duration by two (temporal series; T6, $167 \mathrm{~ms}$, up to T48, $20.8 \mathrm{~ms}$ ) or by dividing the frequency spacing between the tones by two (spectral series; S16, 1/16 of an octave, up to S128, 1/128 of an octave or 4.7 cents). Eight different tone pairs (one rising and one falling, at each of four different frequencies; frequency range, 500-1,000 hertz) were used for each difficulty level. Subjects heard stimuli through headphones binaurally and reported the pitch-change direction by pressing a key.

For all groups, performance was best in the reference condition (ST) and was significantly reduced when either the tone duration (Fig. 1b; 'T' bars) or the frequency difference
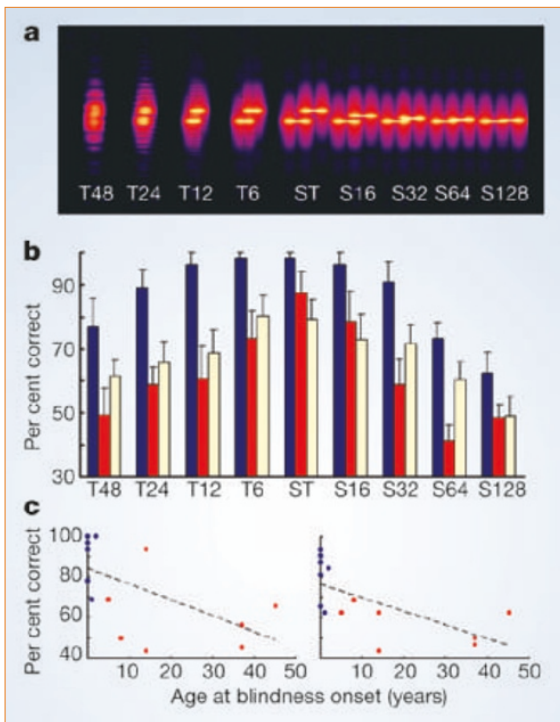

Figure 1 Judgement of direction of pitch change by early-blind, late-blind and sighted participants. a, Spectrogram of examples of the pure tones used in the task for temporal (T) and spectral (S) series; time increases horizontally and frequency increases vertically; colour intensity reflects the energy of the stimuli. Each tone pair shows a representative example of stimuli used in each of the nine conditions, chosen here with a rising pitch from the first to the second tone. The two tones in each pair were always presented in succession; the impression of temporal overlap for the most rapid conditions (temporal series) is an artefact related to the influence of the acoustic uncertainty principle on the timefrequency representation. b, Proportion of correct answers ( \pm s.e.m.) given by early-blind (blue bars), late-blind (red bars) and sighted (white bars) subjects for the nine conditions. c, Significant negative correlation between age of blindness onset and behavioural performance in the pitch-direction task in temporal (left; T6 to T48) and spectral (right; S16 to S128) conditions. Blue and red dots represent early- and late-blind groups, respectively.

between the tones (Fig. 1b; ' $S$ ' bars) was decreased $(P<0.001)$. Early-blind subjects showed significantly better overall performance than either the late-blind or sighted subjects (Fig. 1b; main effect, $P=0.008$; post hoc Tukey: $P=0.009$ and $P=0.026$, respectively). The results followed this pattern for both the temporal $(P=0.011)$ and the spectral $(P=0.011)$ parts of the data set. Performance by the early-blind group for the most rapid condition (T48) was equivalent to that of sighted subjects in the easiest condition (ST), which comprised durations that were greater by an order of magnitude. No significant difference could be detected between late-blind and sighted subjects $(P=0.67)$.

There was a significant negative correlation between age of blindness onset and overall performance (partial correlation $r=-0.65, P=0.012$ ) across the entire group of blind subjects; this relation held even after accounting for the duration of blindness
( $r=-0.49, P=0.045$, one-tailed). Again, this finding held for both the spectral (Fig. 1c, right; $r=-0.65, P=0.011$ ) and the temporal (Fig. 1c, left; $r=-0.60, P=0.024$ ) parts of the data set.

Early-blind subjects were better than both late-blind and sighted subjects at determining the direction of pitch change, for different temporal as well as spectral levels. We conclude that compensatory auditory mechanisms following visual deprivation must extend beyond the spatial domain. Our finding that a large part of the variance (42\%) could be accounted for by the age of blindness onset may explain why conflicting results are found when early- and late-blind subjects are pooled together ${ }^{4,6}$. Moreover, it is in agreement with the idea that cerebral plasticity is more efficient at early developmental stages. Frédéric Gougoux ${ }^{\star}$, Franco Lepore ${ }^{\star}$, Maryse Lassonde*, Patrice Voss ${ }^{\star}$, Robert J. Zatorre $\dagger$, Pascal Belin ${ }^{\star}$

${ }^{\star}$ Centre de Recherche en Neuropsychologie et Cognition, Département de Psychologie, Université de Montréal, CP 6128, Succursale Centre-ville,

Montréal, Québec H3C 3J7, Canada

e-mail: pascal.belin@umontreal.ca

$\dagger$ Montreal Neurological Institute, McGill

University, Montréal, Québec H3A 2B4, Canada

1. Röder, B. et al. Nature 400, 162-166 (1999).

2. Lessard, N., Paré, M., Lassonde, M. \& Lepore, F. Nature 395, 278-280 (1998).

3. Muchnick, C., Efrati, M., Nemeth, E., Malin, M. \& Hildesheimer, M. Scand. Audiol. 20, 19-23 (1991).

4. Sakurabayshi, H., Sato, Y. \& Ueehara, E. J. Psychol. Blind 1, 3-10 (1956).

5. Pitman, D. J. Am. Found. Blind. Res. Bull. 11, 63-79 (1965).

6. Juurma, J. Am. Found. Blind. Res. Bull. 14, 109-122 (1967)

7. Stankov, L. \& Spilsbury, G. Appl. Psychol. Meas. 2, 491-503 (1978).

8. Johnsrude, I. S., Penhune, V. B. \& Zatorre R. J. Brain 123, 155-163 (2000).

9. Zatorre, R. J. \& Belin, P. Cereb. Cortex 11, 946-953 (2001).

Supplementary information accompanies this communication on Nature's website.

Competing financial interests: declared none.

\section{Fisheries \\ Mislabelling of a depleted reef fish}

$\Lambda$ ny fish species that appears to be readily available in the marketplace will create an impression among the public that there is a plentiful supply of that fish in the sea, but this may belie the true state of the fisheries' stock. Here we use molecular genetic analysis to show that some three-quarters of the fish sold in the United States as 'red snapper' - the US Food and Drug Administration's legally designated common name for Lutjanus campechanus ${ }^{1}$ - belong to another species. Mislabelling to this extent not only 
defrauds consumers but could also adversely affect estimates of stock size if it influences the reporting of catch data that are used in fisheries management.

The red snapper, or L. campechanus, is found in offshore waters around coral reefs and rocky outcroppings ${ }^{2}$ and is one of the most economically important fisheries in the Gulf of Mexico, with greater total landings and commanding higher prices than any other snapper species ${ }^{3,4}$. In 1996, the Gulf of Mexico Fishery Management Council and the United States Department of Commerce declared that L. campechanus was grossly overfished and called for strict management measures to restore stocks to sustainable levels.

Tight restrictions may create an economic incentive for seafood substitution, whereby less valuable species are mislabelled and sold under the names of more expensive ones. Substitutions among closely related fish species are difficult to detect, because most distinguishing features are lost during processing. Although the name 'red snapper' is applied to other species in different countries, only L. campechanus can legally be labelled as red snapper in the United States .

To investigate the extent of possible mislabelling in the United States, we did a molecular phylogenetic analysis of fish purchased from nine vendors in eight states (Fig. 1). We compared DNA sequences from retail samples with those deposited in GenBank, which contains sequences from all snappers for which landing data are recorded by the US National Marine Fisheries Service (NMFS).

Our results show that mislabelling is a large-scale phenomenon: $77 \%$ of fish sold as red snapper (17/22) were in fact other species (Fig. 1). This estimate has a margin of error of $17 \%$, meaning that between $60 \%$ and $94 \%$ of fish sold as red snapper in the United States are mislabelled. Among the sequences from fish that are not L. campechanus, five are grouped very closely with those from other Atlantic species. Of these, sequences from two are nearly identical to those from the lane snapper, differing only by singlenucleotide substitutions (Fig. 1). The three other putative Atlantic snappers in our sample are grouped with vermilion snapper, with only about $2 \%$ sequence divergence.

Surprisingly, more than half of our analysed sequences are either grouped closely with species from other regions of the world or represent species too rare to have yet been included in molecular phylogenetic surveys ${ }^{5}$. For example, two fish can be definitively identified as crimson snappers, a species from the Indo-West Pacific. And sequences from several other fish form clades that do not seem to be closely related to any reference sequences. These fish represent species that are either not caught in US waters or not yet individually managed by the NMFS.

Mislabelling of fish can occur at different points in the commercial process: for
Figure 1 Maximum-likelihood tree $(G T R+I+G$ model) of cytochrome- $b$ DNA sequences from retail 'red snapper' (GenBank accession numbers: AY294187205; AY651957-59) and reference sequences (AF239677-78, 80-82; AF240750; AF381270; AF031516; AF299290; U26949, 51-58, 61-62). Numbers at nodes are bootstrap percentages $(1,000$ replicates $)$ from an unweighted parsimony search. Sequences of 953 base pairs were obtained using the polymerase chain reaction and the primers CB12F (5'-TGGCAAGCCTACGCAAAAC-3') and CB13R (5'-TATTCCGCCGATTCAGGTAA-3') followed by automated sequencing on an $\mathrm{ABI}$ 377. Phylogenetic analyses were completed using PAUP* 4.0 software $^{13}$. State abbreviations: $\mathrm{DE}$, Delaware; $\mathrm{FL}$, Florida; LL, Illinois; MA, Massachusetts; NY, New York; NC, North Carolina; SC, South Carolina; WI, Wisconsin.

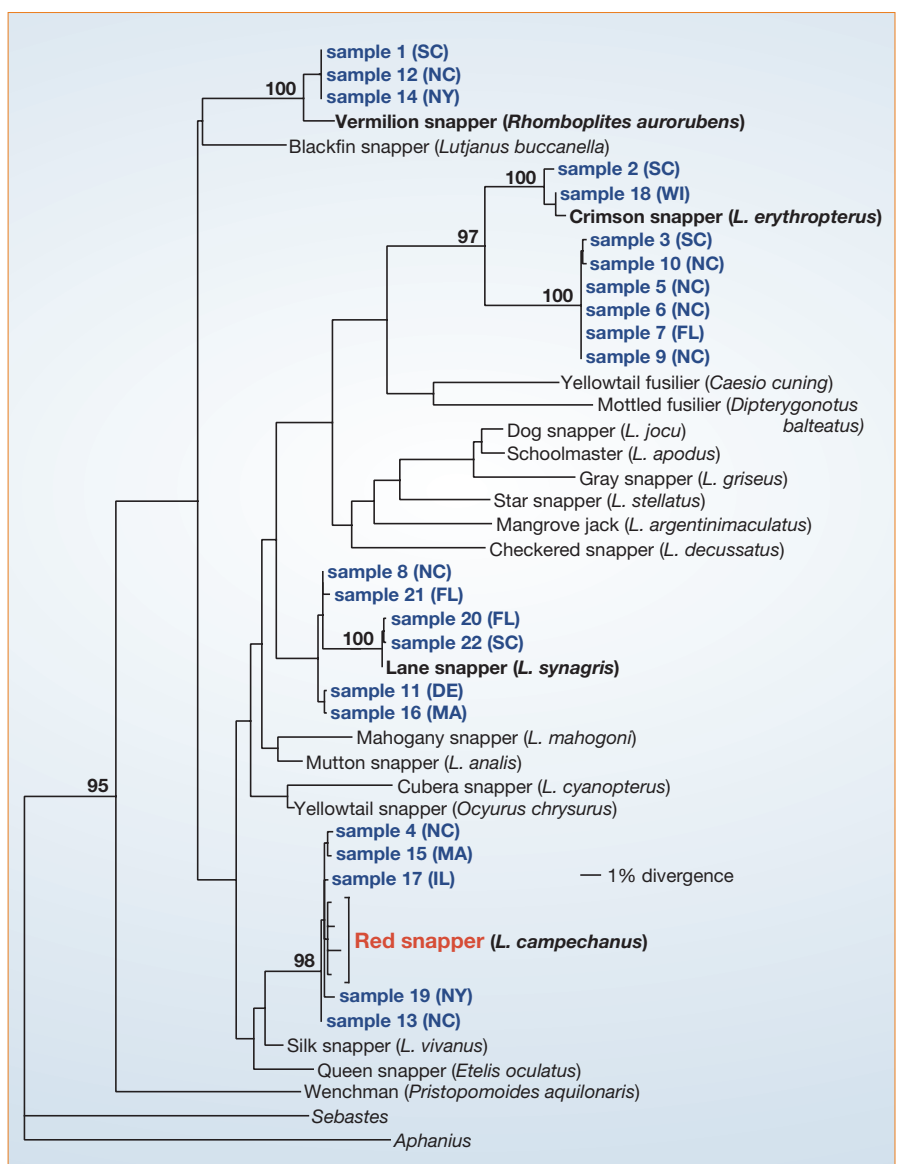

example, retail mislabelling probably explains the presence of crimson snapper in our sample, because these fish occur in an entirely different part of the world from L. campechanus. Fish substitutions may occur on the boat or at the dock, because species that are morphologically similar to L. campechanus are caught together, as is the case for most reef fisheries ${ }^{6}$. The implications for resource management are important: substitutions made before commercial landing data are submitted to the regulatory agencies will cause inflation of catch estimates for the most desirable species and simultaneous underreporting of catches for less desirable, and potentially unmanaged, species. Although it is widely acknowledged that intentional misreporting and unintentional misidentification of species can both bias fishery-dependent data ${ }^{7-11}$, the contribution of this effect cannot be evaluated unless fish are sampled before the collection of catch data.

The remarkable extent of product mislabelling in the case of $L$. campechanus threatens to distort the status of fish stocks as perceived by consumers, which contributes to the false impression that the supply of fish is keeping up with demand ${ }^{12}$.

Peter B. Marko*, Sarah C. Lee*, Amber M. Rice $\dagger$, Joel M. Gramling $\dagger$, Tara M. Fitzhenry $\dagger$, Justin S. McAlister $\dagger$, George R. Harper $\dagger$, Amy L. Moran ${ }^{\star}$ Departments of ${ }^{\star}$ Marine Sciences and $\dagger$ Biology,
University of North Carolina, Chapel Hill, North Carolina 27599-3300, USA

e-mail:pmarko@unc.edu

1. Office of Seafood, Food and Drug Administration (http://www.cfsan.fda.gov/ frf/seaintro.html).

2. Moran, D. Biological report 82(11.83) TR EL-82-4 (National Wetlands Research Center, Louisiana, 1988)

3. Huang, T.-S., Marshall, M. R., Kao, K.-J., Otwell, W. S. \& Wei. C.-I. J. Agric. Food Chem. 43, 2301-2307 (1995).

4. National Marine Fisheries Service, Fisheries Statistics and Economics Division, Silver Spring, Maryland (http://www.st.nmfs.gov/).

5. Sarver, S. K., Freshwater, D. W. \& Walsh, P. J. Copeia 3, 715-719 (1996).

6. Johannes, R. E. Trends Ecol. Evol. 13, 243-246 (1998).

7. Watson, R. \& Pauly, D. Nature 414, 689-695 (2001).

8. Reef Fish Assessment Panel Report (Gulf of Mexico Fishery Management Council, 1994).

9. Marine Fisheries Stock Assessment Improvement Plan (National Marine Fisheries Service, 2001).

10. Atlantic Billfish Research Plan (National Marine Fisheries Service, 2002)

11. Porch, C. E. \& Cass-Calay, S. L. Status of the Vermilion Snapper Fishery in the Gulf of Mexico: Assessment 5.0 SFD-01/02-129 (Southeast Fisheries Science Center, Miami, Florida, 2001).

12. Review of Existing Public Opinion Data on the Oceans (The Ocean Project, 1999).

13. Swofford, D. L. PAUP*: Phylogenetic Analysis Using Parsimony (*and Other Methods), version 4 (Sinauer, Sunderland, Massachusetts, 2001)

Competing financial interests: declared none.

\section{brief communications arising online}

www.nature.com/bca

Ecology: Climate-change effect on Lake Tanganyika? W. W. Eschenbach (doi:10.1038/nature02689)

Reply: C. M. O’Reilly, P.-D. Plisnier, A. S. Cohen, S. R. Alin

(doi:10.1038/nature02737) 